\title{
Regards
}

\section{Les jardins botaniques : entre science et commercialisation}

\author{
Denis Barabé $^{1}$, Alain Cuerrier ${ }^{2}$, Angélique Quilichini ${ }^{3}$ \\ 1 Botaniste, Institut de recherche en biologie végétale, Jardin botanique de Montréal, Université de Montréal, Montréal (Québec), \\ Canada, H1X 2B2 \\ 2 Botaniste, Institut de recherche en biologie végétale, Jardin botanique de Montréal, Université de Montréal, Montréal (Québec), \\ Canada, H1X 2B2 \\ 3 Enseignante-chercheure en écologie, CNRS, UMR8172 Écologie des forêts de Guyane, 97387 Kourou, France
}

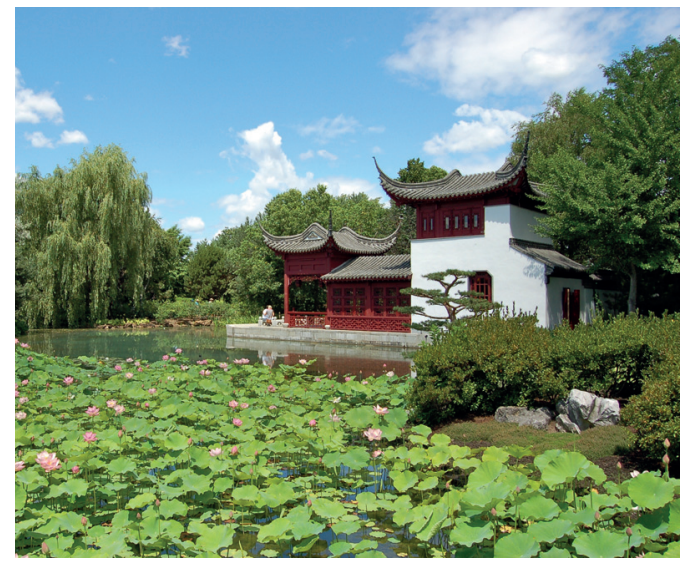

Le jardin chinois du jardin botanique de Montréal (Manuel Menal, CC BY-SA 2.0).
Denis Barabé est professeur associé au département de sciences biologiques de l'Université de Montréal. Il a travaillé pendant plus de trente ans comme botaniste au jardin botanique de Montréal. Ses recherches portent principalement sur la morphologie et la systématique des plantes à fleurs. Alain Cuerrier détient un doctorat en systématique végétale. Après un passage à l'Université du Québec à Montréal comme chargé de cours, il a participé à la mise en place du jardin des Premières-Nations au jardin botanique de Montréal. À partir de 2001, il oriente ses recherches en ethnobotanique et en ethno-écologie. Ses travaux le mènent en territoire nordique où il collabore avec les Cris et les Inuits à propos de leurs savoirs traditionnels et des changements climatiques. Angélique Quilichini possède un doctorat en écologie évolutive. En 2003, elle a été recrutée comme maître de conférences à l'Université Paul Sabatier de Toulouse où elle a participé à la mise en place d'enseignements et de collections au jardin botanique Henri Gaussen. Depuis septembre 2009, elle est accueillie en délégation auprès du CNRS dans l'UMR Ecofog.

\section{Introduction}

Pour la majorité des gens, les jardins botaniques désignent des sites accueillants, agrémentés de platesbandes aux couleurs changeantes selon les saisons. Pour d'autres, plus rares, ils rappellent des institutions à l'architecture austère où sont entreposés des herbiers centenaires, témoins des périodes fructueuses de la taxonomie classique. Bien que la vision des jardins botaniques varie selon les individus, ils sont généralement perçus comme des établissements éducatifs et des lieux de détente, où la science côtoie l'esthétisme. De façon

Auteur correspondant: D. Barabé, denis.barabe@umontreal.ca plus formelle, ils se définissent globalement comme des institutions où l'on conserve des collections de plantes vivantes à des fins éducatives et scientifiques. Cette définition générale offre un cadre précis pour analyser les rôles complémentaires des jardins botaniques, tels que l'éducation, la recherche, le maintien de collections de plantes vivantes et de jardins paysagers, dont l'importance varie selon le type de jardin et le cadre administratif. Près de 2700 institutions qualifiées de jardins botaniques sont recensées dans la base de données «GardenSearch » de l'organisation internationale Botanic Gardens Conservation International $\left(\mathrm{BGCI}^{1}\right)$. D'après les données compilées par Pautasso et Parmentier (2007), il existe environ 700 jardins botaniques proprement dits et

1 http://www.bgci.org. 
plus de 1700 arboretums dans 169 pays différents. La moitié d'entre eux se trouve principalement dans 11 pays des régions tempérées: Australie, Chine, France, Allemagne, Inde, Italie, Japon, Russie, Suisse, Royaume-Uni, États-Unis. Le plus petit occupe une superficie de 0,1 ha et le plus grand, 1681 ha. Le premier fut créé en 1544 à Pise, en Italie. Pourtant, aujourd'hui encore, de nouveaux jardins botaniques voient le jour, l'un des derniers en date étant celui du sultanat d'Oman, de quelque 420 ha.

Nous proposons dans cette synthèse une approche historique et sociétale du rôle des jardins botaniques. Bien que l'origine de ces structures soit assez ancienne, il s'agit toutefois de jeunes institutions si on les compare aux bibliothèques ou aux universités dont l'origine remonte au $\mathrm{XI}^{\mathrm{e}}$ siècle (Université de Bologne, Italie). L'histoire des jardins botaniques publics peut globalement se diviser en quatre grandes périodes, depuis la naissance et l'édification (1544-1770), en passant par le développement et la maturation (1770-1930), l'ouverture au public (1930-1980) et enfin la commercialisation (1980-). Barabé (1991) brosse, dans son étude sur les approches didactiques des jardins botaniques, une histoire qui reflète et repose sur les grandes disciplines, soit la botanique, l'horticulture, l'ethnobotanique et l'architecture de paysage. Cette étude est cependant centrée sur la didactique, alors que nous envisageons ici de revoir cette histoire dans un discours plus général. Chacune des quatre périodes citées ci-dessus correspond donc à des particularités de l'époque, des disciplines scientifiques nouvelles et des approches éducatives différentes qui ont dessiné les grands traits des jardins actuels.

\section{Naissance et édification (1544-1770) : le développement scientifique}

La botanique est pratiquée depuis l'Antiquité mais ce n'est qu'à partir du XVIII ${ }^{\mathrm{e}}$ siècle qu'elle acquiert son statut de science à part entière. Pourtant, les premiers jardins botaniques sont créés dès le milieu du $\mathrm{XVI}^{\mathrm{e}}$ en Italie (Pise, 1544 et Padoue, 1545), suivis par ceux de Breslau (1587), Leyde (1590), Montpellier (1593) et Heidelberg (1593), puis, au XVII ${ }^{\mathrm{e}}$, par ceux d'Oxford (1640) et le « Jardin du Roy » à Paris (ouvert au public en 1650). Dès leur création, ils sont le site de culture de végétaux, notamment de plantes économiques et ornementales, mais ils servent aussi de lieu d'enseignement. Par exemple, les jardins botaniques de Padoue (1545) et de Montpellier (1593) étaient sous la responsabilité des facultés de médecine et regroupaient des collections de plantes médicinales (ou simples) servant à l'apprentissage des futurs médecins. Mais rapidement, les jardins botaniques jouent également un rôle prépondérant dans les progrès de la systématique végétale ainsi que de l'agronomie et de la médecine, suite à l'introduction en culture de nouvelles espèces comestibles ou ayant des propriétés thérapeutiques (Heywood, 2011). Au siècle des Lumières, le nouveau contexte épistémologique permet l'émergence de botanistes professionnels (par exemple, des professeurs, des explorateurs naturalistes) qui contribuent à hisser la botanique au rang de science autonome. Tout comme cette nouvelle science, les jardins botaniques se libèrent progressivement de la tutelle de la médecine au profit d'un nouveau cadre scientifique fondé sur l'anatomie, la morphologie et la systématique végétales. Ils deviennent alors des institutions scientifiques à part entière, notamment entre 1770 et 1930, période pendant laquelle sont publiés des recueils botaniques de référence. Dans cette optique, le jardin des Plantes (spécificité française) de Montpellier (1593) a certainement joué un rôle de précurseur. Un de ses responsables les plus célèbres, Pierre Magnol (1638-1715, intendant de 1694 à 1697), est l'auteur du Prodromus Historiae Generalis Plantarum (1689), où sont inventoriées pour la première fois 76 familles de plantes dont la plupart des descriptions sont encore valides aujourd'hui. Cet aspect scientifique se développe de telle sorte que la décade 1770 représente ce qu'on peut appeler l'âge d'or de la botanique du XVIII ${ }^{\mathrm{e}}$ siècle. En effet, la systématique est alors à son apogée avec la publication de l'Exposition d'un nouvel ordre des plantes, adopté dans les démonstrations $d u$ Jardin royal (1774) d'Antoine-Laurent de Jussieu (17481836), œuvre monumentale qui présente les bases d'un nouveau système de classification des plantes (Stevens, 1994 ; Spary, 2000). De l'autre côté de la Manche, grâce aux efforts de Joseph Bank (1743-1820) et William Aiton (1731-1793), le jardin botanique de Kew connaît un développement exceptionnel et acquiert rapidement une position enviable comme institution scientifique et éducative, étendant son influence un peu partout dans le monde. À la fin du XIXe, le jardin botanique de Berlin a été intimement associé à la publication du Pflanzenfamilien, œuvre essentielle de la systématique (1898), sous la direction de Adolf Engler (1844-1930), alors directeur du jardin. En raison de son importance, cet ouvrage est d'ailleurs de nouveau en cours de réédition ; il en est à sa $13^{\mathrm{e}}$ édition par Borntraeger.

\section{Développement et maturation (1770-1930) : de la botanique systématique à l'horticulture}

À la fin du XVIII e siècle, et surtout, au XIX', l'expansion des colonies, alors sous la tutelle des pays européens, entraîne la création de plusieurs jardins botaniques (Heywood, 2011). Ce fut le cas notamment de celui de Buitenzorg (Bogor, Java) fondé début XIX ${ }^{\mathrm{e}}$ par le gouverneur anglais de l'époque. Rappelons, par ailleurs, que $l^{\prime}$ Inde se classe aujourd'hui parmi les 11 pays qui 
comptent le plus de jardins botaniques au monde et celui de Calcutta (Kolkata) occupe une place de premier rang dans ce pays. Ses fondateurs considéraient qu'il serait profitable à la population de Calcutta et au commerce britannique en permettant, entre autres, l'introduction de plantes en Inde. Dans le même ordre d'idée, le jardin botanique de Saint-Pierre (Martinique), détruit lors de l'éruption de la montagne Pelée, fut un autre bel exemple de la nature des jardins coloniaux. Charles Bélanger (1853-1881), qui en était alors le directeur (Thésée, 2004), écrivait que les buts de ce jardin étaient de : « [...] concourir aux intérêts généraux de la mère patrie en fournissant aux autres colonies et aux établissements de la Métropole toutes les plantes indigènes ou régionales utiles à l'économie rurale ou intéressantes pour l'horticulture [...]». Comme le montrent les photos d'époque, ce jardin comprenait aussi des espaces naturels et des aménagements paysagers ouverts aux visiteurs du dimanche. L'horticulture ornementale et l'architecture de paysage constituent donc des éléments-clés des jardins du XIX ${ }^{\mathrm{e}}$ siècle en exerçant un pouvoir attractif sur le public. D'ailleurs, la présence d'éléments horticoles et architecturaux est souvent une caractéristique des anciens jardins coloniaux. Cela est particulièrement clair dans le jardin d'essai du Hamma, à Alger (1832), où se déroule une superbe allée de Draceana. Il en va de même pour celui de Kisantu (République démocratique du Congo), créé en 1927, où, dès le début, les activités d'introduction et d'acclimatation de nouvelles plantes agricoles ont côtoyé la création d'espaces ornementaux et décoratifs.

Ainsi, bien que les jardins botaniques connaissent un développement scientifique remarquable, les aspects horticoles et esthétiques prennent aussi beaucoup plus d'importance du fait de l'attrait exercé par les plantes exotiques sur les visiteurs. Songeons, par exemple, aux immenses feuilles de Victoria, qui, encore aujourd'hui, sont le centre d'attraction de nombreuses serres de végétaux aquatiques. Ou bien, encore, à la floraison périodique de l'Amorphophallus titanum qui augmente, temporairement, l'affluence dans de nombreux jardins botaniques. La présentation de plantes spectaculaires demeure encore l'un des moyens les plus efficaces d'attirer le public. En fait, ils continuent de jouer, dans une certaine mesure, le rôle de « curiosités » qu'avaient les Cabinets d'histoire naturelle au XVII ${ }^{\mathrm{e}}$ siècle. C'est également le cas, tout récemment, du pin de Wollemi (Wollemia nobilis), à la fois un point d'attraction et un des éléments du discours lié à la conservation ex situ de plantes rares.

\section{Ouverture au public (1930-1980) : éducation, nouvelles attractions...}

Cette période est particulièrement importante dans la mesure où l'attrait des jardins botaniques va s'accentuer amenant ainsi un renouvellement des collections et des activités traditionnelles de manière à attirer un plus grand public. Ainsi, durant les années 1930-1980, nous assistons à un engouement pour tout ce qui touche à l'horticulture ornementale, notamment en Amérique. Cet attrait pour la plante en tant qu' objet esthétique influence les jardins botaniques qui développent alors de plus en plus de collections horticoles et présentent périodiquement des expositions florales sur des thèmes particuliers. $C^{\prime}$ est aussi durant cette période qu'ils mettent en place différents programmes d'éducation du public. Il ne s'agit plus uniquement de présenter aux visiteurs des plantes inusitées et spectaculaires, mais de les renseigner, sinon de les instruire, sur différents aspects du règne végétal (Teuscher, 1940). C'est le cas, par exemple, du jardin des plantes utiles au jardin botanique de Montréal où des écriteaux détaillent l'origine, la taxonomie et l'utilisation de plantes cultivées dans les régions tempérées. Outre la présentation croissante de plantes horticoles, cette ouverture vers le public s'est en effet concrétisée par l'importance donnée à l'aspect éducatif des collections à l'aide de moyens didactiques, restreints, à cette époque, à des informations présentées sous forme de panneaux et de dépliants. Mais l'information éducative ne se limite pas aux plantes utilisées par l'homme. Cette période correspond aussi à la naissance et au développement de l'écologie qui souligne la dynamique fragile des écosystèmes menacés par la croissance de la population humaine. Il n'est donc pas surprenant de voir les jardins botaniques de cette période accorder une place de plus en plus importante à la reconstitution artificielle de formations végétales, mais aussi à la préservation de milieux naturels à l'intérieur de leur enceinte.

Toutefois, malgré l'essor qu'ont connu les jardins botaniques après la Deuxième Guerre mondiale, nombre d'entre eux font face à des difficultés financières, souvent aiguës, à la fin des années 1970. On s'interroge de plus en plus sur leur vocation et même leur utilité et il faut souvent faire preuve d'ingéniosité pour justifier des soutiens financiers reçus de sources publiques ou privées. Les jardins botaniques ne sont pas des institutions financièrement autosuffisantes; leur développement et leur pérennité nécessitent des subventions. Ils subissent de fortes pressions pour augmenter leur rentabilité. Le développement de jardins spécialisés et la réalisation d'attractions particulières s'avèrent donc des solutions idéales pour augmenter le nombre de visiteurs. Ce changement d'orientation marque en un sens le début de la phase commerciale. Il ne suffit plus d'éduquer, il faut aussi pouvoir rentabiliser financièrement les jardins.

Dans les années 1980, l'accès aux jardins botaniques, tels ceux de Kew ou de Montréal, devient payant. Cela permet bien sûr d'augmenter les revenus, mais aussi de développer de nouveaux projets attractifs. Ces projets 
s'orientent d'abord vers l'ambiance asiatique, même si l'attrait exercé par l'Asie dans le domaine de l'horticulture et de l'architecture n'est pas nouveau. $\mathrm{Si}$, dès le XVIII ${ }^{\mathrm{e}}$ siècle, l'architecte Chambers construit une pagode au jardin botanique de Kew, en 1893, le financier Albert Kahn s'installe à Boulogne-sur-Seine et décide d'aménager un jardin japonais sur sa propriété (vers 1910). Il est ouvert au public en 1937 et restauré en 1989. De même, au début du $X X^{\mathrm{e}}$ siècle, le botaniste Ernest Wilson parcourt et photographie la Chine à la recherche de nouvelles plantes horticoles intéressantes. Mais il semble que ce soit surtout à partir des années 1980 que les jardins botaniques aménagent des jardins japonais et chinois d'envergure. Ce type de réalisations fera connaître des plantes et des aménagements paysagers différents, mais aura aussi comme conséquence d'attirer les visiteurs, à une époque où ces institutions doivent diversifier leurs sources de revenus pour garantir un apport financier constant. Ainsi, on peut aussi bien trouver des jardins japonais à Hambourg, qu'à Edmonton, Moscou ou Montréal. De sorte que les jardins botaniques n'ont pas été étrangers à l'engouement des Nord-Américains pour la culture des bonsaïs : celui de Montréal a contribué de façon majeure à populariser les arbres nains en Amérique en présentant au grand public, dans les années 1980, une collection substantielle venue du Japon.

Dans ce contexte, serres et jardins extérieurs deviennent des attractions de plus en plus importantes. Il est impossible d'énumérer ici, ne serait-ce que partiellement, les différentes collections qui y sont conservées. Certaines sont cependant communes à tous les jardins botaniques. La présence de ces grands types de collections est motivée par des choix botaniques, bien sûr, mais aussi par des questions de marketing en vue d'attirer les visiteurs. Depuis l'apparition des premières orangeries, comme celle de Kew en 1761, les jardins botaniques des régions tempérées ont su profiter des serres pour cultiver et propager des végétaux tropicaux qui suscitent encore aujourd'hui la curiosité du grand public. Ces serres ont toujours joué un rôle important dans le maintien de collections de plantes vivantes à des fins esthétiques, éducatives, scientifiques et économiques (Florent et Pautz, 2007 ; Allain, 2010). Dans les jardins botaniques se trouvent fréquemment une serre de végétaux des régions tropicales humides et une serre de cactées et autres plantes grasses des régions arides. Cette dernière est certainement la plus commune. En présentant des végétaux exotiques, souvent peu connus, elle a l'avantage de capter l'attention du public. Il suffit de visiter quelques jardins botaniques pour se rendre compte que les serres de plantes grasses possèdent toutes un air de famille, alors que celles de plantes tropicales peuvent beaucoup varier d'une institution à l'autre, selon le type d'aménagement, selon le matériel disponible ou l'orientation scientifique. Par exemple, au jardin botanique Henri Gaussen (Toulouse), un mur végétal déploie une végétation épiphyte, surplombant les parterres d'herbacées et d'arbustes tropicaux. Certains jardins présentent aussi fréquemment une serre de plantes aquatiques où les immenses feuilles de Victoria côtoient des végétaux semi-aquatiques comme les Colocasia et les Cyrtosperma. Il arrive même que ce type de serre serve d'aquarium comme c'est le cas au jardin botanique d'Édimbourg. À ces trois grands types d'exposition pourront s'en ajouter d'autres dont les thèmes varieront selon l'historique du jardin, les intérêts des conservateurs, les fonds disponibles ou les axes de recherche. Ainsi, on pourra trouver, par exemple, des serres consacrées aux fougères, bonsaïs et Bégoniacées (Montréal), Éricacées (Édimbourg), Aracées (Nancy), Broméliacées (Heidelberg), Orchidées et plantes alpines (Kew).

Les collections extérieures détiennent une grande importance en horticulture, puisqu'elles permettent de connaître la rusticité des végétaux là où ils sont cultivés. Elles contribuent ainsi au développement du commerce horticole et à l'introduction de nouvelles plantes en culture. De même que pour les serres, les mêmes types se retrouvent souvent dans les différents jardins: plantes vivaces, plantes médicinales, plantes comestibles, plantes aquatiques, plantes alpines, roseraies, etc. Le développement de collections particulières dépendra des politiques propres à chacune des institutions. Les petits jardins botaniques font souvent preuve de beaucoup d'imagination dans l'aménagement et le choix de leurs collections extérieures. Par exemple, le jardin botanique d'Oxford possède un fort bel ensemble de variétés d'anciens rosiers. Au jardin botanique de Cambridge, des plantes horticoles sont disposées le long d'un chemin selon leur date d'introduction dans les jardins européens. Cambridge présente aussi un jardin des odeurs où le chemin qui le ceinture descend en dessous du niveau du sol de façon à ce que le visiteur puisse sentir les fleurs odorantes sans avoir à se pencher. Et que dire du jardin botanique Henri Gaussen (Toulouse) où les collections extérieures sont disposées selon une spirale ethnobotanique centrale, aménagée selon l'évolution de l'utilisation des plantes, couvrant les trois-quarts du terrain?

Un autre type de collections, quelquefois présent dans les jardins botaniques, mais qui a aujourd'hui perdu beaucoup de popularité, est la collection systématique, où les plantes sont disposées selon leurs affinités taxonomiques, rappelant ainsi que l'histoire de ces institutions est intimement associée à celle des grands ouvrages de systématique. Il n'est donc pas surprenant que l'on veuille transposer sur le terrain les progrès de cette science. Voilà pourquoi plusieurs jardins botaniques possèdent une collection où les plantes sont disposées selon 
une systématique qui reflète plus ou moins les connaissances botaniques de l'époque. Le jardin botanique de Hambourg a aménagé, dans les années 1970, un jardin systématique représentant la classification de Takhtajan (1910-2009), botaniste soviéto-arménien, où le réseau de chemins relie entre eux les différents ordres. Même le botaniste américain Cronquist (1912-1992), contemporain et ami de Takhtajan, n'est pas en reste, puisque le jardin botanique de Nancy comprend une collection de dicotylédones fondée sur sa classification. De nos jours, ce sont les arbres phylogénétiques de la systématique moléculaire que l'on veut représenter. Au jardin botanique de Kew, la disposition des plantes dans le jardin systématique (Order Beds) suivait, depuis 1869, la classification de G. Bentham (1800-1884) et J.D. Hooker (18171911), qui en était alors le directeur. Afin de tenir compte des développements récents de la systématique, des travaux ont été entrepris depuis quelques années pour représenter, dans cette collection, la nouvelle classification des plantes à fleurs de l'Angiosperm Phylogeny Group (APG, 2009). Bien qu'intéressante d'un point de vue scientifique et didactique, la collection systématique demeure souvent peu attrayante pour le visiteur. De plus, elle occupe beaucoup d'espace tout en étant financièrement coûteuse. Elle ne suscite donc guère $d$ 'intérêt auprès des administrateurs de la plupart des jardins botaniques. En fait, depuis la fin du XX $\mathrm{X}^{\mathrm{e}}$, sauf dans de rares exceptions, le développement de nouvelles collections basées uniquement sur des aspects botaniques n'entre plus dans leurs prérogatives.

\section{Commercialisation et conservation, une nécessité parfois conflictuelle (1980-)}

Vers 1980, on voit apparaître deux nouvelles tendances qui, à première vue, semblent plus ou moins contradictoires mais qui peuvent pourtant évoluer de manière harmonieuse. D'une part, on assiste à une commercialisation de plus en plus grande des jardins botaniques par l'intermédiaire d'événements spéciaux rivalisant d'ingéniosité pour attirer le public. De l'autre, la conservation et la préservation in situ et ex situ des espèces et des biotopes en voie de disparition prend de plus en plus d'importance. Les jardins botaniques ont même été considérés comme des sanctuaires de sauvegarde des espèces en voie de disparition. Un peu comme ces zoos que l'on compare à des arches de Noé. Il s'agit là, bien entendu, d'une vision simpliste, car chacun sait que l'unique moyen efficace d'empêcher la disparition des espèces demeure la protection intégrale de milieux naturels.

Pour assurer le rôle de conservation que seront appelés à jouer les jardins botaniques, le Botanic Gardens Conservation International (BGCI) est créé en 1987. C'est un organisme international dont l'objectif est d'engager les jardins botaniques à préserver les plantes menacées dans l'optique du bien-être des populations humaines (Wyse-Jackson et Sutherland, 2000 ; Dodd et Jones, 2010). Afin d'offrir un cadre précis pour coordonner la conservation des plantes à l'échelle internationale, le programme "Global Strategy for Plant Conservation » (GSPC), est créé, en 2002, sous la tutelle du Secrétariat de la Convention sur la diversité biologique (Williams et al., 2012). Dans cette perspective, beaucoup de jardins botaniques ont pris le tournant de la conservation et ont développé des programmes locaux visant la préservation et la multiplication des espèces végétales menacées d'extinction (Wyse-Jackson et Kennedy, 2009). Par exemple, au Canada, un programme intitulé «Conservation de la diversité des plantes : le défi de 2010 pour les jardins botaniques canadiens » a été mis sur pied en 2006. Ce programme inclut des projets comme la multiplication d'espèces menacées, la réintroduction dans leur milieu naturel d'espèces en voie de disparition, la conservation ex situ des graines d'espèces menacées et des recherches ethnobotaniques avec les communautés amérindiennes (les Premières Nations) pour documenter et préserver les connaissances botaniques traditionnelles. D'ailleurs, les jardins botaniques sont appelés à intervenir davantage dans les recherches en ethnobotanique qui demeurent un élément-clé dans la préservation de la biodiversité mondiale (Cuerrier et Arnason, 2008). Par exemple, au jardin botanique de Montréal, le jardin des PremièresNations, résultat d'une consultation et d'une collaboration avec la majorité des Premières Nations du Québec, soit 11 Nations, vise à faire connaître la flore des principales régions où vivent les Amérindiens du Québec et les utilisations qu'ils en font. La création de cette collection particulière est intimement liée à la mise sur pied d'un nouveau programme de recherche en ethnobotanique. En Afrique, la plupart des jardins sont avant tout des aires protégées. C'est notamment le cas du jardin botanique de Limbé, au Cameroun (créé en 1892), qui est aujourd'hui une destination écotouristique courue. Il abrite de plus un centre international pour la recherche en biodiversité, concernant la conservation de la forêt naturelle et de plantes médicinales, ainsi qu'un centre de formation pour les populations locales. De même, le jardin botanique d'Aburi, au Ghana, participe à l'éducation à l'usage traditionnel des plantes et à leur conservation via la mise en place de jardins particuliers. Enfin, les activités privilégiées par le réseau de jardins botaniques africains concernent notamment l'éducation à l'environnement et, surtout, l'éducation aux pratiques culturales traditionnelles des plantes médicinales (ABGN, 2006). De manière générale, les jardins botaniques situés dans les régions tropicales sont beaucoup plus orientés vers la conservation des plantes dans leurs milieux naturels que ne le sont ceux des régions tempérées (Chen et al., 2009). 
Le rôle de conservation, que les jardins ont toujours joué, est maintenant exacerbé par les politiques de sauvegarde de la biodiversité (Delmas et al., 2011) et de restauration des habitats dégradés. Cet objectif va modeler les activités éducatives qui s'orientent maintenant vers une sensibilisation à l'environnement et au développement durable.

Toutefois, et malgré leurs importances, ces aspects de conservation du patrimoine ne suffisent pas, il faut trouver d'autres solutions pour attirer le visiteur. Il devient nécessaire de renouveler l'image des jardins botaniques et, surtout, de trouver de nouvelles sources de revenus pour les financer alors que leur entretien et leur fonctionnement sont de plus en plus coûteux. Afin de répondre à ces besoins, des expositions ou des événements spéciaux relatifs aux plantes sont organisés. La récurrence annuelle de ces animations constitue une source importante de revenus. Au jardin botanique de Montréal, par exemple, trois grands événements attirent chaque année des milliers de visiteurs. Dès septembre, « La Magie des Lanternes », dans l'enceinte du jardin chinois, permet de découvrir tout un monde de lanternes représentant différents personnages ou animaux. Entièrement faites de soie colorée, elles sont dessinées à Montréal puis réalisées minutieusement par des artisans de Shanghai. En octobre, l'exposition «Le Grand Bal des Citrouilles » prend place. Il y a, bien entendu, le traditionnel concours de la plus grosse citrouille, mais aussi un concours de citrouilles décorées et une pièce de théâtre (Pépo-Citrouille) pour les jeunes écoliers. En hiver, l'exposition « Papillons en Liberté » permet de découvrir de magnifiques papillons du monde entier. Dans la grande serre, des milliers de chrysalides se métamorphosent sous l'œil émerveillé des visiteurs. Ainsi, les serres dont l'unique fonction était auparavant la présentation de plantes tropicales se transforment pour plusieurs semaines par an en volière pour les papillons.

Il faut cependant considérer l'aspect négatif de la commercialisation. Ce «changement de paradigme» peut réduire l'intérêt au sein des jardins botaniques pour des éléments moins porteurs, tels que la systématique ou le maintien de collections intéressantes du point de vue botanique, mais dont les taxa demeurent d'un engouement limité pour un public qui cherche la plante vedette. Les jardins font face au défi qu'engendre cette double nécessité de l'ouverture au public et de l'exigence scientifique, tout en comptant avec les contraintes économiques (Lieutaghi et Musset, 2008). Ils doivent donc rester ouverts et créatifs face aux nouveaux enjeux sans toutefois oublier leurs missions premières. Cela constitue leur défi : multiplier les expositions et les événements novateurs afin d'attirer une clientèle toujours plus importante et, à la fois, maintenir les anciens objectifs et remplir les nouveaux objectifs que la problématique environnementale a soulevés, notamment le rôle que les jardins botaniques doivent ou peuvent jouer quant aux changements climatiques (Donaldson, 2009 ; Heywood, 2011 ; Schulman et Lehvävirta, 2011).

\section{Le constat actuel}

Les jardins botaniques remplissent donc une gamme de fonctions complémentaires. Voilà pourquoi ils représentent une richesse culturelle et scientifique pour l'avenir. Jusqu'à maintenant, leur structure et leur développement s'articulaient autour de thèmes complémentaires dont l'importance variait selon le type de jardin : l'éducation (botanique, horticulture, développement durable), la recherche (systématique, physiologie, écologie), la conservation et la détente (parc, architecture de paysage). Cependant, ces fonctions et usages traditionnels sont aujourd'hui modifiés pour répondre à des exigences de rentabilité où le visiteur devient un client. Rappelons que durant $l^{\prime}$ histoire des jardins botaniques, à partir du moment où ils ont été ouverts au public, l'augmentation du nombre de visiteurs a toujours été une préoccupation des gestionnaires. Cependant, si dans le passé on suscitait la curiosité du public par le caractère exotique ou esthétique de nombreuses plantes, aujourd'hui on utilise des moyens (spectacles, jeux de lumières, réceptions, etc.) qui sont quelquefois fort éloignés du monde végétal, transformant ainsi la définition classique de jardin botanique ou, du moins, diminuant son importance.

Actuellement, l'éducation occupe une place de plus en plus grande. Nombreux sont les jardins qui ont développé récemment des programmes éducatifs pour les groupes scolaires, allant de l'élémentaire à l'Université. C'est le cas, par exemple, du jardin botanique de Toulouse, structure universitaire dont le foncier est géré par la municipalité. Les collections sont sous la responsabilité du personnel scientifique de l'Université et le Muséum d'histoire naturelle de Toulouse s'occupe de l'accès au public. Le défi a donc été d'attirer son attention sur un petit espace, qui ne doit pas apparaître seulement comme un lieu de détente après la visite du Muséum. Le jardin extérieur se déploie autour d'une collection centrale dédiée à l'ethnobotanique. Le public, ainsi attiré vers les plantes par une approche ethnobotanique (notamment par les interactions des végétaux avec les autres organismes vivants), tisse un lien avec la recherche scientifique. Toutefois, comme le soulignaient Lieutaghi et Musset (2008), " La plante elle-même ne raconte pas ses rapports avec les sociétés, il lui faut des traducteurs", d'où l'importance de développer des approches didactiques fortes.

Les collections vivantes sont beaucoup plus qu'un simple objet éducatif ou esthétique ; elles constituent aussi un réservoir de phénomènes biologiques et un immense stock de matériel génétique. Toutefois, la mise en 
valeur de leur potentiel scientifique constitue certainement un aspect important du développement scientifique d'une région. Dans ce contexte, les jardins botaniques s'efforcent de mettre en valeur leurs collections en les rendant facilement disponibles. Cependant, ils doivent user de prudence et appliquer une éthique stricte à propos de l'accès à leurs collections, comme le stipule le document The CBD for Botanists (Williams et al., 2006).

Comme mentionné précédemment, la recherche a toujours été un élément primordial dans l'évolution des jardins botaniques qui demeurent, encore aujourd'hui, un endroit-clé pour les travaux en systématique et en conservation (Morris, 2006; Crane et al., 2009). Par exemple, c'est dans l'enceinte du jardin botanique de Montréal que vient d'être construit le nouveau Centre de la biodiversité de l'Université de Montréal qui met à la disposition des chercheurs des instruments à la fine pointe de la technologie. De façon plus précise, les collections servent, entre autres, dans les études morphologiques, systématiques, phytochimiques ou physiologiques qui nécessitent la comparaison de plantes venant de différents milieux (Barabé, 2008 ; 2010).

La recherche peut concerner le domaine horticole dans des programmes d'introduction de nouvelles espèces ou cultivars, la sélection d'hybrides ou la propagation de plantes particulières. Au jardin botanique de Nancy, par exemple, il existe un programme de recherche sur la multiplication des anciennes obtentions horticoles lorraines (lilas, pivoines, fuchsias...) et sur la conception d'un verger-conservatoire pour les anciennes variétés fruitières locales. Le jardin botanique peut aussi servir de lieu d'innovation en architecture de paysage comme en témoignent le récent jardin botanique de Bordeaux ou l'aménagement de la collection ethnobotanique du jardin Henri Gaussen (Toulouse). Il peut même être un laboratoire pour la conception et l'expérimentation de nouveaux concepts de serre comme c'est le cas de l'Alpine House du jardin botanique de Kew.

Cependant, avec les problèmes environnementaux actuels, d'autres défis attendent les jardins botaniques. Selon certains scientifiques, la restauration des écosystèmes dégradés demeure l'une des priorités pour l'humanité au XXI ${ }^{\mathrm{e}}$ siècle (Hardwick et al., 2011). Or, plusieurs activités traditionnelles des jardins botaniques sont importantes pour atteindre cet objectif. Toutefois, malgré le lien étroit entre ces ressources et les besoins de la restauration écologique, peu d'institutions sont engagées dans cette voie. À cet effet, Hardwick et al. (2011) proposent d'augmenter de façon significative les activités de restauration réalisées dans les jardins botaniques en modifiant légèrement leurs objectifs et missions. Par exemple, même un petit jardin botanique pourrait développer des protocoles techniques pour la régénération des plants nécessaires à la restauration (Hardwick et al., 2011). Or, à notre avis, un tel changement $d^{\prime}$ orientation ne peut se faire sans tenir compte des objectifs particuliers de chaque jardin botanique. De plus, une telle problématique doit être soutenue par une approche didactique visant à sensibiliser le public. C'est d'ailleurs pourquoi le jardin botanique de Montréal mettra en scène un jardin des phytotechnologies afin de faire connaître aux visiteurs les recherches en cours dans ce domaine.

\section{Et demain?}

Bien entendu, la situation d'un jardin botanique doit être analysée en le replaçant dans son contexte historique, administratif, géographique et politique. Il va de soi que ce contexte impose une contrainte sur le mode d'organisation et la dynamique dans lequel évoluera ce jardin. Bien que la recherche, l'éducation, l'horticulture, la détente et la conservation soient inhérentes à ce type d'institution, l'un ou l'autre de ces éléments sera privilégié selon l'orientation donnée. Toutefois, un jardin botanique qui veut suivre l'air du temps tout en gardant ses orientations scientifiques et éducatives ne peut se développer harmonieusement sans tenir compte des besoins et des attentes des visiteurs, sans mettre sur pied des programmes éducatifs adéquats et sans être ouvert aux activités de recherches intensives et aux problèmes environnementaux qui s'abattent actuellement sur la planète.

Ainsi, dans le contexte du développement durable, l'ethnobotanique constitue certainement une activité scientifique et culturelle d'avenir pour les jardins botaniques. Ils participent d'ailleurs déjà au développement - que celui-ci soit tacite ou voulu - de cette discipline. Il faut revenir sur la raison première de l'existence des jardins pour bien saisir la relation entre le monde végétal et l'homme dans ce qu'il a de plus fragile : sa santé, voire sa survie. En effet, les premiers jardins inaugurent le lien entre simples et maladies, entre - plus tard, il est vrai plantes alimentaires et cultures. Ils existent parce que l'homme a maintenu un dialogue avec les plantes. Et n'est-ce pas ce qui définit l'ethnobotanique? Dans un temps où la biodiversité est en péril, il va de soi que la diversité culturelle qui s'y rattache doit être mise en valeur (Martin, 2004 ; Métailié, 2008). Ce dialogue entre l'homme et les plantes est la raison de la présence des visiteurs au sein même des jardins, comme il caractérise le travail des ethnobotanistes. Plantes vivantes, plantes séchées des herbiers, qui souvent ou parfois cohabitent avec les jardins, cartes, livres et bibliothèques, provoquent et alimentent le discours ethnobotanique de façon vivante et directe. Les jardins ont cette chance de ne pas être enclavés dans la réalité du bâti comme c'est le cas des universités. Aussi, l'esprit de l'ethnobotanique s'unit à celui des jardins botaniques : ce n'est pas tant un retour qu'une continuation des grands thèmes de ces institutions. En 2007, des chercheurs ont signé la déclaration de Kau'î qui stipule l'importance de cette discipline dans le 
contexte de la perte de la biodiversité (Prance et al., 2007). Ainsi, écrivent-ils :

«The strong links between biological and cultural diversity uniquely position ethnobotany to help us craft effective local solutions to many of the global issues that confront us as a species. Some of the most challenging of these issues are food security, deforestation, pollution, the maintenance of human health, the quality of human life, and resource depletion of all kinds. »

Ce rôle est essentiel pour les pays en développement où la médecine repose avant tout sur le savoir traditionnel (déclaration de Beijing de l'OMS ; Atiti, 2011). Toutefois, l'intérêt grandissant pour la médecine traditionnelle, en particulier pour les plantes médicinales, fait réapparaître un point sensible : les droits de propriété intellectuelle. Plusieurs groupes de recherche, liés ou non à des entreprises pharmaceutiques privées, misent sur la découverte de nouveaux principes actifs contre le cancer, le diabète ou toute autre maladie, à partir des données ethnobotaniques (Lewis, 2003 ; Cuerrier et al., 2012). C'est pourquoi plusieurs jardins botaniques et muséums se sont dotés d'une politique d'accession à leurs collections (c'est le cas du Muséum national d'histoire naturelle à Paris et du jardin botanique de Montréal). En 2006, le jardin botanique de Kew a publié The CBD for Botanists (Williams et al., 2006) qui exhorte les jardins à se munir de principes en accord avec ceux de la Convention sur la diversité biologique et notamment, avec son $3^{\mathrm{e}}$ objectif, l'accès aux ressources génétiques et le partage juste et équitable des avantages liés à leur utilisation (APA) décliné dans le protocole de Nagoya (Secretariat of the Convention on Biological Diversity, 2011). Sur le plan international, des associations, comme l'International Society of Ethnobiology (2006), cherchent aussi à sensibiliser les chercheurs et les populations locales au travail et au commerce éthiques lorsqu'ils touchent à la biodiversité. Les collections des jardins botaniques ont donc besoin de mesures de protection, d'autant plus qu'elles hébergent des plantes parfois rapportées d'études ethnobotaniques et pour lesquelles des populations autochtones ou locales possèdent des droits ancestraux. Ce sont là de nouveaux défis pour les jardins botaniques à travers le monde.

Mais tous les jardins botaniques doivent-ils pour autant réorienter leurs objectifs en fonction des problématiques environnementales actuelles? Nous ne croyons pas qu'il soit possible d'énoncer des lignes de conduite générales applicables à tous. Chaque jardin botanique a une histoire, un cadre financier et des objectifs prioritaires qui lui sont propres. Et bien que les problèmes environnementaux actuels demeurent une priorité, seuls ceux soutenant de grandes équipes de recherches sont en mesure d'apporter une contribution scientifique significative dans ce domaine. D'un autre côté, il semble évident que le rôle des jardins est appelé à se transformer sous l'influence des contraintes, non pas uniquement scientifiques et éducatives, mais aussi politiques et administratives. Mais est-il trop utopique de penser comme Soderstrom (2001, p. 216) :

«Gardens lovers should also insist that botanical gardens be financed by all of us, largely through our taxes. Botanical gardens must continue to catalogue and safeguard the earth's wealth of plants and to lay the ground work through conservation education for larger changes required to sustain this green earth [...] They should be beautiful and interesting, attracting people of every age from every part of society, but they should not have to compete head to head with amusement parks for income for admission to survive. And, perhaps especially, they should not be left vulnerable to influence by whatever organization or individual pays the bills. »

\section{Références}

ABGN, 2006. African Botanic Gardens Network Bulletin, $\mathrm{n}^{\circ} 11$. Allain, Y.-M., 2010. De l'orangerie au palais de cristal, une histoire des serres, Versailles, Quæ.

Angiosperm Phylogeny Group, 2009. An update of the Angiosperm Phylogeny Group classification for the orders and families of flowering plants, Botanical Journal of the Linnean Society, 161, 105-121.

Atiti, A.B., 2011. The Role of Botanic Gardens in the Dissemination of Ethnobotanical Knowledge in Kenya (http:// www.bgci.org/education/1769/).

Barabé, D., 1991. Les approches didactiques et le jardin botanique, Musées, 13, 3, 150-159.

Barabé, D., 2008. The use of the Montreal Botanical Garden's Aroid collection for research, IAS Newsletter (International Aroid Society), 30, 4, 2-4.

Barabé, D., 2010. Les collections de plantes vivantes du jardin botanique de Montréal: un outil essentiel pour la recherche, Naturaliste Canadien, 134, 2, 4-7.

Chen, J., Cannon, C.H., Hu, H., 2009. Tropical botanical gardens: at the in situ ecosystem management frontier, Trends in Plant Science, 14, 584-589.

Crane, P.R., Hopper, S.D., Raven, P.H., Stevenson, D.W., 2009. Plant science research in botanic gardens, Trends in Plant Science, 14, 575-577.

Cuerrier, A., Arnason, J.T., 2008. Introduction. Ethnobotany in Canada: where biological and cultural diversity meet, Botany, 86, v-vi.

Cuerrier, A., Downing, A., Patterson, E., Haddad, P., 2012. Aboriginal antidiabetic plant project with the James Bay Cree of Québec: An insightful collaboration, Journal of Enterprising Communities: People and Places in the Global Economy, 6, 3, 251-270.

Delmas, M., Larpin, D., Haevermans, T., 2011. Rethinking the links between systematic studies and ex situ living collections as a contribution to the Global Strategy for Plant Conservation, Biodiversity and Conservation, 20, 287-294.

Dodd, J., Jones, C., 2010. Redefining the role of Botanic gardenTowards a new social purpose, Research Centre for Museums and Galleries, University of Leicester \& BGCI, Leicester. 
Donaldson, J.S., 2009. Botanic gardens science for conservation and global change, Trends in Plant Science, 14, 608-613.

Florent, F., Pautz, F., 2007. Serres des jardins botaniques d'Europe, Avignon, Aubanel.

Hardwick, K.A., Fiedler, P., Lee, L.C. et al., 2011. The role of botanic gardens in the science and practice of ecological restoration, Conservation Biology, 25, 265-275.

Heywood, V.H., 2011. The role of botanic gardens as resource and introduction centres in the face of global change, Biodiversity and Conservation, 20, 221-239.

International Society of Ethnobiology, 2006. International Society of Ethnobiology Code of Ethics (with 2008 additions), http://ethnobiology.net/code-of-ethics/.

Lewis, W.S., 2003. Pharmaceutical discoveries based on ethnobotanical plants: 1985 to 2000 and beyond, Economic Botany, 57, 126-134.

Lieutaghi, P., Musset, D., 2008. Introduction : Genèse d'un colloque, in Lieutaghi, P., Musset, D. (Eds), Jardins et médiation des savoirs en ethnobotanique, Actes du colloque de Salagon, Forcalquier, Musée de Salagon/Éditions C'est-àdire, $7-12$.

Martin, G., 2004. Ethnobotany: A Method Manual, London, Earthscan.

Métailié, G., 2008. De l'ethnobotanique à ses jardins, in Lieutaghi, P., Musset, D. (Eds), Jardins et médiation des savoirs en ethnobotanique, Actes du colloque de Salagon, Forcalquier, Musée de Salagon/Éditions C'est-à-dire, 21-28.

Morris, E., 2006. Gardens in full bloom, Nature, 440, 860-863.

Pautasso, M., Parmentier, I., 2007. Are the living collections of the world's botanical gardens following species-richness patterns observed in natural ecosystems? Botanica Helvetica, 117, 15-28.

Prance, G.T., Raven, P.H., Wichman, C. et al., 2007. Ethnobotany: the science of survival, Economic Botany, 61, 1-2.
Schulman, L., Lehvävirta, S., 2011. Botanic gardens in the age of climate change, Biodiversity and Conservation, 20, 217-220.

Secretariat of the Convention on Biological Diversity, 2011. Nagoya Protocol on Access to Genetic Resources and the Fair and Equitable Sharing of Benefits Arising from their Utilization to the Convention on Biological Diversity: text and annex. Montreal, United Nations Environmental Programme.

Spary, E.C., 2000. Utopia's Garden: French Natural History from Old Regime to Revolution, Chicago, The University of Chicago Press.

Soderstrom, M., 2001. Recreating Eden. A Natural History of Botanical Gardens, Montréal, Canada, Vehicule Press.

Stevens, P.F., 1994. The Development of Biological Systematics: Antoine-Laurent de Jussieu, Nature, and the Natural System, New-York, Columbia University Press.

Teuscher, H., 1940. Programme d'un jardin botanique idéal, Montréal, Mémoires du Jardin botanique de Montréal, $\mathrm{n}^{\circ} 1$.

Thésée, F., 2004. Le Jardin botanique de Saint-Pierre-Martinique (1803 - 1902), Paris, Éditions Caribéennes.

Williams, C., Davis, K., Cheyne, P., 2006. The CBD for Botanists: An introduction to the Convention on Biological Diversity for people working with botanical collections, version 2, Kew, The Board of Trustees, Royal Botanic Gardens.

Williams, S.J., Jones, J.P.G., Clubbe, C., Sharrock, S., Gibbons, J.M., 2012. Why are some biodiversity implemented and others ignored? Lessons from the uptake of the global strategy for plant conservation by botanic gardens, Biodiversity and Conservation, 21, 175-187.

Wyse Jackson, P.S., Kennedy, K., 2009. The Global Strategy for Plant Conservation: a challenge and opportunity for the international community, Trends in Plant Science, 14, 578-580.

Wyse Jackson, P.S., Sutherland, L.A., 2000. International Agenda for Botanic Gardens in Conservation, U.K., Botanic Gardens Conservation International. 